\title{
Selection Indices Studies in Maize (Zea mays L.) under Multiple Abiotic Stress Conditions
}

\author{
Preeti Massey $^{1^{*}}$, S. S. Verma ${ }^{2}$ and M. Z. K. Warsi ${ }^{3}$
}

Dept. of Genetics and Plant Breeding, College of Agriculture, GBPUA\&T, Pantnagar, Uttarakhand (263 145), India

\section{Article History}

Manuscript No. AR1401c

Received in $11^{\text {th }}$ July, 2015

Received in revised form $14^{\text {th }}$ March, 2016

Accepted in final form $25^{\text {th }}$ June, 2016

\section{Correspondence to}

*E-mail: preeti.genetics@gmail.com

\section{Keywords}

Abiotic stress, low-N, waterlogging, selection index, economic weight

\begin{abstract}
The present investigation was undertaken on maize (Zea mays L.) under two abiotic stress conditions viz. low-nitrogen (low soil fertility) and excess soil moisture conditions (waterlogging) during kharif 2010. Studies were carried out for developing suitable selection indices in relation to multiple abiotic stress tolerance. The experimental material consisted of twelve lines and four testers and their 48 single crosses planted in Randomized Block Design. Analysis of variance revealed significance for all the traits studied in both the conditions, indicating there by the existence of high genetic variability in the genotypes. In low-N conditions, the best performing line was $\mathrm{L}_{5}$ followed by and among testers $\mathrm{T}_{3}$ ranked highest. Among the crosses, $\mathrm{L}_{11} \times \mathrm{T}_{4}$ ranked highest followed by $\mathrm{L}_{8} \times \mathrm{T}_{1}$ and $\mathrm{L}_{11} \times \mathrm{T}_{3}$. In waterlogging conditions, $\mathrm{L}_{6}$ performed better followed by $\mathrm{L}_{5}$. Among the crosses $\mathrm{L}_{6} \times \mathrm{T}_{4}$ ranked highest. Economic weights were assigned to various traits under low-nitrogen and excess soil moisture (ESM) conditions. Among the crosses, aggregate score were higher in most of the cases but general trend was that ESM traits had the lower score values. Crosses $\mathrm{L}_{11} \times \mathrm{T}_{4}, \mathrm{~L}_{8} \times \mathrm{T}_{1}$, $\mathrm{L}_{11} \times \mathrm{T}_{3}, \mathrm{~L}_{7} \times \mathrm{T}_{2}$ and $\mathrm{L}_{6} \times \mathrm{T}_{2}$ performed well in low-nitrogen conditions for the assigned selection criteria. In waterlogging trials, $\mathrm{L}_{6} \times \mathrm{T}_{4}, \mathrm{~L}_{7} \times \mathrm{T}_{2}$ and $\mathrm{L}_{4} \times \mathrm{T}_{1}$ performed excellent while crosses $\mathrm{L}_{5} \times \mathrm{T}_{1}, \mathrm{~L}_{12} \times \mathrm{T}_{2}, \mathrm{~L}_{5} \times \mathrm{T}_{4}$, and $\mathrm{L}_{3} \times \mathrm{T}_{3}$ ranked very low in ESM trials. Thus for ESM tolerance, the crosses $\mathrm{L}_{6} \times \mathrm{T}_{4}$ and $\mathrm{L}_{7} \times \mathrm{T}_{2}$ are the best available crosses according to the given selection criteria and for low-nitrogen tolerance, $\mathrm{L}_{11} \times \mathrm{T}_{4}$ and $\mathrm{L}_{8} \times \mathrm{T}_{1}$ were the best crosses.
\end{abstract}

\section{Introduction}

Crowned with the rhetoric phrase the queen of cereal maize (Zea mays L.) is the world's most important cereal and occupies the apex position in terms of production at the world level. It is an economically important cereal crop for human food, animal feed, fiber and a range of industrial uses. Maize crop grown in tropics during summer-rainy season occasionally face extreme climatic conditions and various biotic or abiotic stresses that severely limit crop growth and development and eventually yield potential. Among the abiotic stresses, low nitrogen and Excess Soil Moisture (ESM) are the most important constraints for maize production and productivity in the world and Asian region. Indian soils have been characterized as being low in organic matter and N. Tropical rain fed soils in semi-arid regions are typically low in organic matter, often containing less than $1 \%$ organic matter, with a total $\mathrm{N}$ generally not exceeding $0.1 \%$. About $77 \%$ of these rain fed soils are classified as low to very low in available N (Katyal et al., 1994). Recent statistics on $\mathrm{N}$ fertilizer consumption pattern showed that average application of $\mathrm{N}$ in developed countries is $250 \mathrm{~kg} \mathrm{ha}^{-1}$, while in developing countries it is $82 \mathrm{~kg} \mathrm{ha}^{-1}$, and in Sub-Saharan African countries it is as low as $5.0 \mathrm{~kg} \mathrm{ha}^{-1}$ (SAA, 2002). The trend of low $\mathrm{N}$ application contributes to low maize yield of about 1-2 $\mathrm{t} \mathrm{ha}^{-1}$ in developing countries (CIMMYT, 1994), which is in stark contrasts to yields reported from research stations in the same countries ranging from 4-12 $\mathrm{t} \mathrm{ha}^{-1}$ (CIMMYT, 1995, 1996). Yields in Africa are considerably lower than the world average because the cultivation of maize is often prone to low soil fertility (primarily $\mathrm{N}$ but also $\mathrm{P}$ and other nutrient deficiencies) in addition to biotic stresses (FAO, 2010). Additional reasons for low-N fertility in the tropics include poor soil types with low $\mathrm{N}$ mineralization, high run off or leaching of applied fertilizer with heavy rainfall due to light textured soils, and poor uptake of applied $\mathrm{N}$ due to problems of water stress.

In India, excess soil moisture caused by flooding, waterlogging, high water table or heavy soil texture is also one of the most important constraints for crop production where about $8.5 \mathrm{mha}$ of arable land is in the grip of this problem. In 
case of June planting it may coincide with flowering which may interfere with the normal pollination behavior and seed setting (Savita et al., 2004). In India out of total $6.6 \mathrm{mha}$ area of maize over $2.5 \mathrm{mha}$ is prone to face excessive soil moisture/ waterlogging conditions, which causes on average $25-30 \%$ loss of national maize production almost every year (DMR, 2001). The maize crop suffers badly whenever it encounters temporary excess soil moisture (ESM) conditions during the monsoon season or grown in poorly drained converted paddy fields after a rainy season rice crop, a practice more common in Thailand, Japan and Eastern China (Shimizu, 1992). Maize plants are injured more and greater yield losses occur when flooded at early stages. Therefore, it would be desirable to develop maize cultivars with increased resistance to low $\mathrm{N}$ and ESM conditions which is desirable for sustainable production systems with improved yield, without any threat on environment and ecosystems.

Population improvement of a crop is the primary objective of a plant-breeding program. However, the progress in any breeding program depends primarily upon the genetic diversity and the effectiveness of the selection procedure involved. Besides other selection method used for the Population improvement, selection indices are considered as an aid to the breeder for simultaneous selection of multiple traits (Smith, 1936). This tool can help the breeder in spotting the desirable genotype/family of a crop species in a population improvement program. Smith (1936) and Hazel (1943) illustrated the procedure for constructing a selection index that gives maximum advance from selection. Selection indices provide useful information about which traits to be combined (Banziger and Lafitte, 1997). Selection indices have been an effective selection criterion to increase grain yield in corn (Modaressi et al., 2004). Many other Researchers have used selection indices as an effective selection criterion in their breeding programs on different crops (Vikram and Roy, 2003; Xie et al., 1998; Dolan et al., 1996). Keeping this in mind, the proposed study was carried out with the objective to construct selection indices with regard to low $\mathrm{N}$ and ESM tolerance.

\section{Materials and Methods}

The present investigation was undertaken with twelve inbred lines and four testers which were initially screened for their performance in low-N and ESM tolerance and normal conditions at the Crop Research Center of Govind Ballabh Pant University of Agriculture and Technology, Pantnagar, Uttarakhand, India. These inbred lines were then crossed in linextester fashion at Pantnagar to generate $\mathrm{F}_{1}{ }^{\prime} \mathrm{s}$. The experimental material thus consisted of twelve lines, four testers and $48 \mathrm{~F}_{1 ' s}$. Field trials were laid down in low-N, excess soil moisture and normal conditions during kharif
2010 at Pantnagar. The details of the parental lines have been indicated in the Table 1 (Table 1: Details of the parental lines).

\begin{tabular}{|c|c|c|}
\hline Parents & Pedigree & Coded pedigree \\
\hline 1. & POB. $33 \mathrm{C}_{3}-12-2-1-1-2-2$ & $\mathrm{~L}_{1}$ \\
\hline 2. & POB. $33 \mathrm{C}_{3}-12-2-1-2-2-5$ & $\mathrm{~L}_{2}$ \\
\hline 3. & POB. $33 \mathrm{C}_{3}-142-1-6-1-1-4$ & $\mathrm{~L}_{3}$ \\
\hline 4. & POB. $45 \mathrm{C}_{8}-86-1-3-7-6-4$ & $\mathrm{~L}_{4}$ \\
\hline 5. & POB. $45 \mathrm{C}_{8}-45-2-6-1-2-7$ & $\mathrm{~L}_{5}$ \\
\hline 6. & POB. $45 \mathrm{C}_{8}-269-2-4-6-3-3$ & $\mathrm{~L}_{6}$ \\
\hline 7. & POB. $45 \mathrm{C}_{8}-86-1-1-7-5-1$ & $\mathrm{~L}_{7}$ \\
\hline 8. & CLG 1708-1-1-9 & $\mathrm{L}_{8}$ \\
\hline 9. & POB. $45 \mathrm{C}_{8}-45-2-6-1-1-1$ & $\mathrm{~L}_{9}$ \\
\hline 10. & POB. $45 \mathrm{C}_{8}-86-1-3-4-5-2$ & $\mathrm{~L}_{10}$ \\
\hline 11. & POB. $45 \mathrm{C}_{8}-86-1-3-2-2-5$ & $\mathrm{~L}_{11}$ \\
\hline 12. & POB. $45 \mathrm{C}_{8}-269-2-4-6-6-1$ & $\mathrm{~L}_{12}$ \\
\hline 13. & POB. $445 \otimes 58-6-3-B-B-B$ & $\mathrm{~T}_{1}$ \\
\hline 14. & POB. 446-74-2-B-B-B & $\mathrm{T}_{2}$ \\
\hline 15. & CML-421 & $\mathrm{T}_{3}$ \\
\hline 16. & CML-423 & $\mathrm{T}_{4}$ \\
\hline
\end{tabular}

For the present study, parents along with all the developed generations were grown in Randomized Block Design in one row plot of $5 \mathrm{~m}$ length with 3 replications following spacing of $75 \mathrm{~cm}$ between rows and $25 \mathrm{~cm}$ between plants. The crop management practices were applied in the three environments as per the recommendations. In low $\mathrm{N}$ condition, $40 \mathrm{~kg} \mathrm{~N} \mathrm{ha}^{-1}$ was applied. In ESM trials, waterlogging treatment was given at knee high growth stage for 6 days, by keeping continuous submergence with an average depth of ponding of about 5 $\mathrm{cm}$. After 6 days of ponding, water was drained out of the plots. The data were recorded on days to $50 \%$ tasseling, days to $50 \%$ silking, anthesis, silking interval, plant height, ear height, cob length, cob diameter, 100-kernel weight, grain yield, nodes bearing adventitious roots, leaf senescence and number of ears per plant. Appropriate statistical and biometrical analysis for selection index (Smith-Hazel) was carried out. A selection index with many traits is likely to have low heritability (Bernardo and Yu, 2007). Genotypic and phenotypic variance-covariance matrices were developed to aid calculation of index coefficients. Estimated indices were calculated by the method described by Smith (1936).

Application of discriminate function as a basis for making selection on several characters simultaneously is aimed at discriminating the desirable genotypes from undesirable genotypes on the basis of their phenotypic performance. 
Later on, Hazel (1943) developed a simultaneous selection model following path analysis approach.

Smith (1936) defined the genetic worth of an individual (H) as:

$$
H=a_{1} G+a_{2} G_{2}+\ldots \ldots+a_{n} G_{n}=\sum_{\mathrm{i}=1}^{\mathrm{n}} \mathrm{a}_{\mathrm{i}} \mathrm{G}_{\mathrm{i}}
$$

Where, $G_{l}, G_{2}, G_{n}$ are the genotypic (breeding) values of individual characters and $a_{1}, a_{2}, \ldots$. , an signify their relative economic importance. Another function (I) based on the phenotypic performance of various characters, is defined as:

$$
I=b_{1} p_{1}+b_{2} p_{2}+\ldots \ldots \ldots . .+b_{n} p_{n}
$$

Where, $b_{1}, b_{2}, \ldots . ., b_{n}$ are to be estimated such that the correlation between $\mathrm{H}$ and I, i.e. $\mathrm{r}(\mathrm{H}, \mathrm{I})$, becomes maximum. Once such function is obtained, discrimination of good genotypes from the undesirable ones will be possible on the basis of phenotypic performance, i.e. $p_{l}, p_{2}, \ldots . p_{n}$ directly.

The maximization of $\mathrm{r}(\mathrm{H}, \mathrm{I})$ leads to a set of simultaneous equation which upon solving give the desired estimate of $b_{i}$ values.

Considering 4 characters, the simultaneous equations look like as follows:

$b_{1} \times 11+b_{2} \times 12+b_{3} \times 13+b_{4} \times 14=a_{1} G_{11}+a_{2} G_{12}+a_{3} G_{13}+a_{4} G_{14}$ $b_{1} \times 21+b_{2} \times 22+b_{3} \times 33+b_{4} \times 24=a_{1} G_{21}+a_{2} G_{22}+a_{3} G_{23}+a_{4} G_{24}$ $b_{1} \times 31+b_{2} \times 32+b_{3} \times 33+b_{4} \times 34=a_{1} G_{31}+a_{2} G_{32}+a_{3} G_{33}+a_{4} G_{34}$ $b_{1} \times 41+b_{2} \times 42+b_{3} \times 43+b_{4} \times 44=a_{1} G_{41}+a_{2} G_{42}+a_{3} G_{43}+a_{4} G_{44}$ Which in matrix form become :

$$
\left[\begin{array}{llll}
x_{11} & x_{12} & x_{13} & x_{14} \\
x_{21} & x_{22} & x_{23} & x_{23} \\
x_{31} & x_{32} & x_{33} & x_{33} \\
x_{41} & x_{42} & x_{43} & x_{43}
\end{array}\right] \cdot\left[\begin{array}{c}
b_{1} \\
b_{2} \\
b_{3} \\
b_{4}
\end{array}\right]=\left[\begin{array}{llll}
G_{11} & G_{12} & G_{13} & G_{14} \\
G_{21} & G_{22} & G_{23} & G_{24} \\
G_{31} & G_{32} & G_{33} & G_{34} \\
G_{41} & G_{42} & G_{43} & G_{44}
\end{array}\right] \cdot\left[\begin{array}{c}
a_{1} \\
a_{2} \\
a_{3} \\
a_{4}
\end{array}\right]
$$

The solution of these equations gives the estimates of $b_{i}$ values in the following manner :

$b=X^{-1} G a$

Where, $b$ is the column vector, $X^{-1}$ is the inverse of phenotypic variance and covariance matrix and $a$ is the column vector for economic weights.

The various steps involved in the construction of selection indices are described below:

\section{(i) Estimation of variance and covariance matrix}

Genotypic and phenotypic variances and covariance's were determined using Randomized Complete Block Design with analysis of variance for each trait and covariance between every pairs of traits.
The expected mean sums of squares are as follows:

$$
\begin{aligned}
& \mathrm{E}\left(\mathrm{MS}_{\mathrm{g}}\right)=\sigma_{2}^{2}+r \sigma_{\mathrm{g}}^{2} \\
& \mathrm{E}\left(\mathrm{MS}_{\mathrm{e}}\right)=\sigma_{\mathrm{e}}^{2}
\end{aligned}
$$

And, therefore, $\quad \sigma_{g}^{2}=\frac{\mathrm{MS}_{\mathrm{g}}-\mathrm{MS}_{\mathrm{e}}}{r}$, where $\mathrm{r}$ is number of replications.

Thus, the estimated genotypic variance is $\sigma_{g}^{2}$ and the environmental variance is $\sigma_{e}^{2}$. The phenotypic variance, $\sigma_{p}^{2}$, is calculated as $\sigma_{g}^{2}+\sigma_{e}^{2}$.

Similarly, expectations of mean sum of products are calculated as follows:

$$
\begin{aligned}
& E\left(M S P_{g}\right)=\sigma e_{1} e_{2}+r \sigma g_{1} g_{2} \\
& E\left(M S P_{e}\right)=\sigma e_{1} e_{2},
\end{aligned}
$$

And hence, $\sigma g_{1} g_{2}=\frac{M S P_{g}-M S P_{e}}{r}$

Thus, the estimated genotypic covariance is $\sigma g_{1} g_{2}$, and the environmental covariance is $\sigma e_{1} e_{2}$. The phenotypic covariance, $\sigma p_{1} p_{2}$ is calculated as $\sigma g_{1} g_{2}+\sigma e_{1} e_{2}$. The genotypic variance and covariance matrix is constructed as follows:

$\left[\begin{array}{llll}\sigma_{g 1}^{2} & \sigma_{g l g 2} & \sigma_{g l g 3} & \sigma_{g l g 4} \\ \sigma_{g l g 2} & \sigma_{g 2}^{2} & \sigma_{g 2 g 3} & \sigma_{g 2 g 4} \\ \sigma_{g l g 3} & \sigma_{g 2 g 3} & \sigma_{g 3}^{2} & \sigma_{g 3 g 4} \\ \sigma_{g l g 4} & \sigma_{g 2 g 4} & \sigma_{g 3 g 4} & \sigma_{g 4}^{2}\end{array}\right]$

And genotypic variance and covariance matrix is constructed as follows:

$$
\left[\begin{array}{llll}
\sigma_{p 1}^{2} & \sigma_{p l p 2} & \sigma_{p l p 3} & \sigma_{p l p 4} \\
\sigma_{p l p 2} & \sigma_{p 2}^{2} & \sigma_{p 2 p 3} & \sigma_{p 2 p 4} \\
\sigma_{p l p 3} & \sigma_{p 2 p 3} & \sigma_{p 3}^{2} & \sigma_{p 3 p 4} \\
\sigma_{p l p 4} & \sigma_{p 2 p 4} & \sigma_{p 3 p 4} & \sigma_{p 4}^{2}
\end{array}\right]
$$

\section{(ii) Setting of simultaneous equations}

Assuming that all characters are economically equally important, $\mathrm{a}_{1}=\mathrm{a}_{2}=\mathrm{a}_{3}=\mathrm{a}_{4}=1$, we can write down the simultaneous equation (in a matrix form) by using the values given in above two matrices.

$\left[\begin{array}{cccc}\sigma_{p 1}^{2} & \sigma_{p l p 2} & \sigma_{p l p 3} & \sigma_{p l p 4} \\ \sigma_{p l p 2} & \sigma_{p 2}^{2} & \sigma_{p 2 p 3} & \sigma_{p 2 p 4} \\ \sigma_{p l p 3} & \sigma_{p 2 p 3} & \sigma_{p 3}^{2} & \sigma_{p 3 p 4} \\ \sigma_{p l p 4} & \sigma_{p 2 p 4} & \sigma_{p 3 p 4} & \sigma_{p 4}^{2}\end{array}\right]\left[\begin{array}{c}b_{1} \\ b_{2} \\ b_{3} \\ b_{4}\end{array}\right]=\left[\begin{array}{cccc}\sigma_{g 1}^{2} & \sigma_{g l g 2} & \sigma_{g l g 3} & \sigma_{g l g 4} \\ \sigma_{g l g 2} & \sigma_{g 2}^{2} & \sigma_{g 2 g 3} & \sigma_{g 2 g 4} \\ \sigma_{g l g 3} & \sigma_{g 2 g 3} & \sigma_{g 3}^{2} & \sigma_{g 3 g 4} \\ \sigma_{g l g 4} & \sigma_{g 2 g 4} & \sigma_{g 3 g 4} & \sigma_{g 4}^{2}\end{array}\right]\left[\begin{array}{l}1 \\ 1 \\ 1 \\ 1\end{array}\right]$

(iii) The solution of the equation for $b_{i}$ values

As we know: $b=X^{-1} G a$

We first require to inverse the phenotypic variance-covariance matrix. Now the inverse $\left(X^{-I}\right)$ is multiplied with the genotypic 
variance and covariance matrix, i.e. $G$. The resultant matrix i.e. $X^{-1} G$ is multiplied by a vector $a_{i}$ to get $b_{i}$ values.

(iv) The selection index and selection criterion

The mathematical description of the function (I) is known as selection index.

$I=b_{1} p_{1}+b_{2} p_{2}+\ldots .,+b_{n} p_{n}$

Using this function, the selection criterion or index value for each individual is determined as follows:

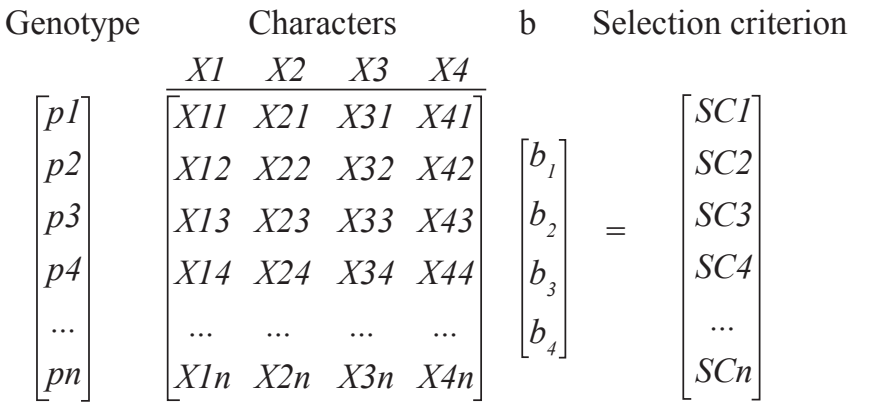

\section{Results and Discussion}

Analysis of variance for all the characters were carried out and it was found that mean squares for all the genotypes studied were highly significant under normal, low-N and ESM conditions, clearly indicating the existence of genetic variability in the genotypes. In normal conditions, among parents, $\mathrm{L}_{5}$ ranked first and $\mathrm{L}_{9}$ ranked last among lines and in case of testers $T_{1}$ ranked first and $T_{4}$ ranked last. In low-N conditions, $\mathrm{L}_{5}$ performed better followed by $\mathrm{L}_{2}$ and the least rank was shown by $\mathrm{L}_{9}$ in case of lines and in testers $\mathrm{T}_{3}$ ranked highest while $\mathrm{T}_{4}$ ranked last as indicated in Table 2 (Table 2: Selection criterion and ranking of parents and crosses in maize for four traits under normal, high $\mathrm{N}$ and low-N conditions). Among the crosses, $\mathrm{L}_{11} \times \mathrm{T}_{4}$ ranked highest followed by $\mathrm{L}_{8} \times \mathrm{T}_{1}$ and $\mathrm{L}_{11} \times \mathrm{T}_{3}$ and the least performing cross for this particular selection index was $\mathrm{L}_{5} \times \mathrm{T}_{4}$. In ESM conditions, $\mathrm{L}_{6}$ performed better followed by $\mathrm{L}_{5}$ and the least rank was shown by $\mathrm{L}_{9}$ in case of lines as indicated in Table 3 (Table 3: Selection

Table 2 : Selection criterion and ranking of parents and crosses in maize for four traits under normal (high N) and low-N conditions

\begin{tabular}{|c|c|c|c|c|c|c|c|c|c|}
\hline \multirow{3}{*}{$\begin{array}{l}\text { Variable / } \\
\text { character }\end{array}$} & \multicolumn{4}{|c|}{$\beta \mathrm{i}$ value } & \multirow{3}{*}{$\begin{array}{l}\text { Variable / } \\
\text { character }\end{array}$} & \multicolumn{4}{|c|}{$\beta \mathrm{i}$ value } \\
\hline & \multicolumn{2}{|c|}{ normal } & \multicolumn{2}{|c|}{ low-N } & & \multicolumn{2}{|c|}{ normal } & \multicolumn{2}{|c|}{ low-N } \\
\hline & score & rank & score & rank & & score & rank & score & rank \\
\hline $\mathrm{L}_{1}$ & 8849.18 & 62 & 6622.45 & 61 & $\mathrm{~L}_{5} \mathrm{~T}_{1}$ & 20494.14 & 40 & 20491.50 & 27 \\
\hline $\mathrm{L}_{2}$ & 11902.97 & 59 & 16698.69 & 47 & $\mathrm{~L}_{5} \mathrm{~T}_{2}$ & 24227.27 & 15 & 24200.58 & 6 \\
\hline $\mathrm{L}_{3}$ & 7327.56 & 63 & 7102.92 & 60 & $\mathrm{~L}_{5} \mathrm{~T}_{3}$ & 25050.14 & 10 & 23955.92 & 10 \\
\hline $\mathrm{L}_{4}$ & 13421.64 & 56 & 8809.45 & 57 & $\mathrm{~L}_{5} \mathrm{~T}_{4}$ & 14619.18 & 55 & 10470.03 & 55 \\
\hline $\mathrm{L}_{5}$ & 20932.03 & 36 & 16728.90 & 46 & $\mathrm{~L}_{6} \mathrm{~T}_{1}$ & 19114.48 & 46 & 16503.29 & 48 \\
\hline $\mathrm{L}_{6}$ & 16314.75 & 52 & 15150.55 & 51 & $\mathrm{~L}_{6} \mathrm{~T}_{2}$ & 26356.79 & 6 & 24581.19 & 5 \\
\hline $\mathrm{L}_{7}$ & 12749.13 & 57 & 6551.71 & 62 & $\mathrm{~L}_{6} \mathrm{~T}_{3}$ & 24695.18 & 12 & 24184.04 & 7 \\
\hline $\mathrm{L}_{8}$ & 9338.65 & 60 & 6062.84 & 63 & $\mathrm{~L}_{6} \mathrm{~T}_{4}$ & 23211.62 & 24 & 22421.66 & 17 \\
\hline $\mathrm{L}_{9}$ & 5047.73 & 64 & 1275.16 & 64 & $\mathrm{~L}_{7} \mathrm{~T}_{1}$ & 20532.68 & 39 & 13517.20 & 52 \\
\hline $\mathrm{L}_{10}$ & 15869.99 & 53 & 12327.51 & 53 & $\mathrm{~L}_{7} \mathrm{~T}_{2}$ & 26785.70 & 4 & 26442.40 & 4 \\
\hline $\mathrm{L}_{11}$ & 16702.96 & 51 & 7832.50 & 59 & $\mathrm{~L}_{7} \mathrm{~T}_{3}$ & 22729.83 & 26 & 18789.70 & 35 \\
\hline $\mathrm{L}_{12}$ & 12087.18 & 58 & 8505.43 & 58 & $\mathrm{~L}_{7} \mathrm{~T}_{4}$ & 25187.81 & 9 & 21260.09 & 23 \\
\hline $\mathrm{T}_{1}$ & 22535.39 & 27 & 19543.95 & 32 & $\mathrm{~L}_{8} \mathrm{~T}_{1}$ & 26564.27 & 5 & 26868.71 & 2 \\
\hline $\mathrm{T}_{2}$ & 18819.55 & 47 & 17558.67 & 42 & $\mathrm{~L}_{8} \mathrm{~T}_{2}$ & 20884.16 & 37 & 17813.30 & 40 \\
\hline $\mathrm{T}_{3}$ & 21065.57 & 33 & 20886.25 & 26 & $\mathrm{~L}_{8} \mathrm{~T}_{3}$ & 22244.48 & 29 & 19923.14 & 45 \\
\hline $\mathrm{T}_{4}$ & 9147.12 & 61 & 8899.67 & 56 & $\mathrm{~L}_{8} \mathrm{~T}_{4}$ & 23030.11 & 25 & 22386.47 & 18 \\
\hline $\mathrm{L}_{1} \mathrm{~T}_{1}$ & 21696.24 & 30 & 20309.81 & 28 & $\mathrm{~L}_{9} \mathrm{~T}_{1}$ & 24492.03 & 13 & 18147.27 & 38 \\
\hline $\mathrm{L}_{1} \mathrm{~T}_{2}$ & 24417 & 14 & 21888.40 & 20 & $\mathrm{~L}_{9} \mathrm{~T}_{2}$ & 23692.94 & 20 & 23333.35 & 14 \\
\hline $\mathrm{L}_{1} \mathrm{~T}_{3}$ & 28386.52 & 3 & 24063.96 & 9 & $\mathrm{~L}_{9} \mathrm{~T}_{3}$ & 20991.55 & 35 & 19993.80 & 30 \\
\hline $\mathrm{L}_{1} \mathrm{~T}_{4}$ & 19472.11 & 44 & 19912.76 & 31 & $\mathrm{~L}_{9} \mathrm{~T}_{4}$ & 19815.38 & 43 & 18561.58 & 37 \\
\hline $\mathrm{L}_{2} \mathrm{~T}_{1}$ & 18017.78 & 50 & 17340.87 & 43 & $\mathrm{~L}_{10} \mathrm{~T}_{1}$ & 23503.16 & 22 & 23416.53 & 13 \\
\hline $\mathrm{L}_{2} \mathrm{~T}_{2}$ & 20142.32 & 42 & 15982.90 & 49 & $\mathrm{~L}_{10} \mathrm{~T}_{2}$ & 21366.22 & 31 & 20931.92 & 25 \\
\hline
\end{tabular}




\begin{tabular}{|c|c|c|c|c|c|c|c|c|c|}
\hline \multirow{3}{*}{$\begin{array}{l}\text { Variable / } \\
\text { character }\end{array}$} & \multicolumn{4}{|c|}{$\beta$ i value } & \multirow{3}{*}{$\begin{array}{l}\text { Variable / } \\
\text { character }\end{array}$} & \multicolumn{4}{|c|}{$\beta$ i value } \\
\hline & \multicolumn{2}{|c|}{ normal } & \multicolumn{2}{|c|}{ low-N } & & \multicolumn{2}{|c|}{ normal } & \multicolumn{2}{|c|}{ low-N } \\
\hline & score & rank & score & rank & & score & rank & score & rank \\
\hline $\mathrm{L}_{2} \mathrm{~T}_{3}$ & 20353.96 & 41 & 19242.79 & 33 & $\mathrm{~L}_{10} \mathrm{~T}_{3}$ & 24699.01 & 11 & 24130.03 & 8 \\
\hline $\mathrm{L}_{2} \mathrm{~T}_{4}$ & 24191.44 & 16 & 23647.40 & 12 & $\mathrm{~L}_{10} \mathrm{~T}_{4}$ & 25357.74 & 8 & 21583.88 & 21 \\
\hline $\mathrm{L}_{3} \mathrm{~T}_{1}$ & 22523.35 & 28 & 21503.89 & 22 & $\mathrm{~L}_{11} \mathrm{~T}_{1}$ & 23300.98 & 23 & 18919.04 & 34 \\
\hline $\mathrm{L}_{3} \mathrm{~T}_{2}$ & 19214.70 & 45 & 18767.49 & 36 & $\mathrm{~L}_{11} \mathrm{~T}_{2}$ & 21270.54 & 32 & 17976.47 & 39 \\
\hline $\mathrm{L}_{3} \mathrm{~T}_{3}$ & 18447.71 & 48 & 15158.63 & 50 & $\mathrm{~L}_{11} \mathrm{~T}_{3}$ & 28683.45 & 2 & 26610.25 & 3 \\
\hline $\mathrm{L}_{3} \mathrm{~T}_{4}$ & 23682.85 & 21 & 22918.61 & 15 & $\mathrm{~L}_{11} \mathrm{~T}_{4}$ & 32176.19 & 1 & 27387.26 & 1 \\
\hline $\mathrm{L}_{4} \mathrm{~T}_{1}$ & 25470.92 & 7 & 22797.26 & 16 & $\mathrm{~L}_{12} \mathrm{~T}_{1}$ & 18179.58 & 49 & 17696.19 & 41 \\
\hline $\mathrm{L}_{4} \mathrm{~T}_{2}$ & 20737.84 & 38 & 21213.44 & 24 & $\mathrm{~L}_{12} \mathrm{~T}_{2}$ & 14797.99 & 54 & 11219.38 & 54 \\
\hline $\mathrm{L}_{4} \mathrm{~T}_{3}$ & 21034.69 & 34 & 17170.95 & 44 & $\mathrm{~L}_{12} \mathrm{~T}_{3}$ & 24028.84 & 19 & 20309.17 & 29 \\
\hline $\mathrm{L}_{4} \mathrm{~T}_{4}$ & 24088.85 & 17 & 23797.00 & 11 & $\mathrm{~L}_{12} \mathrm{~T}_{4}$ & 24057.94 & 18 & 22213.98 & 19 \\
\hline
\end{tabular}

Table 3: Selection criterion and ranking of parents and crosses in maize for ten traits under normal and ESM conditions

\begin{tabular}{|c|c|c|c|c|c|c|c|c|c|}
\hline \multirow{3}{*}{$\begin{array}{l}\text { Variable / } \\
\text { character }\end{array}$} & \multicolumn{4}{|c|}{$\beta$ i value } & \multirow{3}{*}{$\begin{array}{l}\text { Variable / } \\
\text { character }\end{array}$} & \multicolumn{4}{|c|}{$\beta$ i value } \\
\hline & \multicolumn{2}{|c|}{ normal } & \multicolumn{2}{|c|}{ low-N* } & & \multicolumn{2}{|c|}{ normal } & \multicolumn{2}{|c|}{ low-N* } \\
\hline & score & rank & score & rank & & score & rank & score & rank \\
\hline $\mathrm{L}_{1}$ & 3346.19 & 62 & 1367.91 & 60 & $\mathrm{~L}_{5} \mathrm{~T}_{1}$ & 7141.33 & 40 & 829.601 & 63 \\
\hline $\mathrm{L}_{2}$ & 3983.94 & 59 & 3389.20 & 52 & $\mathrm{~L}_{5} \mathrm{~T}_{2}$ & 8583.03 & 16 & 5853.49 & 19 \\
\hline $\mathrm{L}_{3}$ & 2543.73 & 63 & 1342.96 & 61 & $\mathrm{~L}_{5} \mathrm{~T}_{3}$ & 8698.80 & 9 & 6110.88 & 12 \\
\hline $\mathrm{L}_{4}$ & 4763.65 & 56 & 3924.67 & 42 & $\mathrm{~L}_{5} \mathrm{~T}_{4}$ & 5255.76 & 55 & 1757.92 & 59 \\
\hline $\mathrm{L}_{5}$ & 7126.36 & 41 & 4229.90 & 40 & $\mathrm{~L}_{6} \mathrm{~T}_{1}$ & 6754.48 & 46 & 4772.63 & 31 \\
\hline $\mathrm{L}_{6}$ & 5922.43 & 51 & 4469.16 & 35 & $\mathrm{~L}_{6} \mathrm{~T}_{2}$ & 9009.48 & 6 & 6742.36 & 5 \\
\hline $\mathrm{L}_{7}$ & 4699.29 & 57 & 2627.95 & 55 & $\mathrm{~L}_{6} \mathrm{~T}_{3}$ & 8291.04 & 17 & 6480.97 & 9 \\
\hline $\mathrm{L}_{8}$ & 3513.99 & 60 & 2173.47 & 56 & $\mathrm{~L}_{6} \mathrm{~T}_{4}$ & 8157.46 & 22 & 7763.04 & 1 \\
\hline $\mathrm{L}_{9}$ & 2108.32 & 64 & 540.42 & 64 & $\mathrm{~L}_{7} \mathrm{~T}_{1}$ & 7856.38 & 29 & 3614.23 & 49 \\
\hline $\mathrm{L}_{10}$ & 5713.92 & 53 & 3965.73 & 41 & $\mathrm{~L}_{7} \mathrm{~T}_{2}$ & 9074.43 & 4 & 7248.57 & 2 \\
\hline $\mathrm{L}_{11}$ & 5835.42 & 52 & 2112.85 & 57 & $\mathrm{~L}_{7} \mathrm{~T}_{3}$ & 7875.79 & 28 & 4370.82 & 37 \\
\hline $\mathrm{L}_{12}$ & 4282.29 & 58 & 1903.35 & 58 & $\mathrm{~L}_{7} \mathrm{~T}_{4}$ & 8526.91 & 10 & 6060.50 & 13 \\
\hline $\mathrm{T}_{1}$ & 8086.28 & 26 & 6546.67 & 8 & $\mathrm{~L}_{8} \mathrm{~T}_{1}$ & 9021.61 & 5 & 6026.82 & 16 \\
\hline $\mathrm{T}_{2}$ & 6403.47 & 50 & 5236.52 & 27 & $\mathrm{~L}_{8} \mathrm{~T}_{2}$ & 7315.56 & 38 & 5799.06 & 20 \\
\hline $\mathrm{T}_{3}$ & 7321.12 & 37 & 6971.58 & 4 & $\mathrm{~L}_{8} \mathrm{~T}_{3}$ & 7749.57 & 30 & 3731.47 & 46 \\
\hline $\mathrm{T}_{4}$ & 3489.73 & 61 & 6117.76 & 11 & $\mathrm{~L}_{8} \mathrm{~T}_{4}$ & 8093.24 & 24 & 5241.24 & 26 \\
\hline $\mathrm{L}_{1} \mathrm{~T}_{1}$ & 7570.80 & 31 & 5331.36 & 25 & $\mathrm{~L}_{9} \mathrm{~T}_{1}$ & 8417.63 & 13 & 6038.15 & 15 \\
\hline $\mathrm{L}_{1} \mathrm{~T}_{2}$ & 8477.53 & 12 & 5402.93 & 22 & $\mathrm{~L}_{9} \mathrm{~T}_{2}$ & 8164.11 & 21 & 6203.02 & 10 \\
\hline $\mathrm{L}_{1} \mathrm{~T}_{3}$ & 9710.50 & 3 & 5396.58 & 23 & $\mathrm{~L}_{9} \mathrm{~T}_{3}$ & 7416.92 & 35 & 4270.82 & 39 \\
\hline $\mathrm{L}_{1} \mathrm{~T}_{4}$ & 6894.50 & 43 & 4976.63 & 29 & $\mathrm{~L}_{9} \mathrm{~T}_{4}$ & 6876.76 & 44 & 4283.38 & 38 \\
\hline $\mathrm{L}_{2} \mathrm{~T}_{1}$ & 6447.36 & 49 & 3472.04 & 51 & $\mathrm{~L}_{10} \mathrm{~T}_{1}$ & 8213.88 & 19 & 6614.17 & 7 \\
\hline $\mathrm{L}_{2} \mathrm{~T}_{2}$ & 7462.63 & 33 & 3327.55 & 53 & $\mathrm{~L}_{10} \mathrm{~T}_{2}$ & 7337.47 & 36 & 3702.33 & 47 \\
\hline $\mathrm{L}_{2} \mathrm{~T}_{3}$ & 7091.92 & 42 & 3478.18 & 50 & $\mathrm{~L}_{10} \mathrm{~T}_{3}$ & 8512.62 & 11 & 5031.58 & 28 \\
\hline $\mathrm{L}_{2} \mathrm{~T}_{4}$ & 8415.32 & 14 & 4475 & 34 & $\mathrm{~L}_{10} \mathrm{~T}_{4}$ & 8791.91 & 7 & 6005.61 & 17 \\
\hline $\mathrm{L}_{3} \mathrm{~T}_{1}$ & 7934.82 & 27 & 4613.34 & 32 & $\mathrm{~L}_{11} \mathrm{~T}_{1}$ & 8176.15 & 20 & 6052.27 & 13 \\
\hline $\mathrm{L}_{3} \mathrm{~T}_{2}$ & 6816.85 & 45 & 5357.07 & 24 & $\mathrm{~L}_{11} \mathrm{~T}_{2}$ & 7485.62 & 32 & 3828.57 & 44 \\
\hline
\end{tabular}




\begin{tabular}{|c|c|c|c|c|c|c|c|c|c|}
\hline \multirow{3}{*}{$\begin{array}{l}\text { Variable / } \\
\text { character }\end{array}$} & \multicolumn{4}{|c|}{$\beta$ i value } & \multirow{3}{*}{$\begin{array}{l}\text { Variable / } \\
\text { character }\end{array}$} & \multicolumn{4}{|c|}{$\beta$ i value } \\
\hline & \multicolumn{2}{|c|}{ normal } & \multicolumn{2}{|c|}{ low-N* } & & \multicolumn{2}{|c|}{ normal } & \multicolumn{2}{|c|}{ low-N* } \\
\hline & score & rank & score & rank & & score & rank & score & rank \\
\hline $\mathrm{L}_{3} \mathrm{~T}_{3}$ & 6598.16 & 47 & 2992.24 & 54 & $\mathrm{~L}_{11} \mathrm{~T}_{3}$ & 9834.54 & 2 & 5720.87 & 21 \\
\hline $\mathrm{L}_{3} \mathrm{~T}_{4}$ & 8153.21 & 23 & 4475.00 & 33 & $\mathrm{~L}_{11} \mathrm{~T}_{4}$ & 10735.98 & 1 & 6005.60 & 18 \\
\hline $\mathrm{L}_{4} \mathrm{~T}_{1}$ & 8706.99 & 8 & 7071.64 & 3 & $\mathrm{~L}_{12} \mathrm{~T}_{1}$ & 6498.81 & 48 & 3911.99 & 43 \\
\hline $\mathrm{L}_{4} \mathrm{~T}_{2}$ & 7289.06 & 39 & 3688.21 & 48 & $\mathrm{~L}_{12} \mathrm{~T}_{2}$ & 5444.21 & 54 & 1042.60 & 62 \\
\hline $\mathrm{L}_{4} \mathrm{~T}_{3}$ & 7430.32 & 34 & 378.79 & 45 & $\mathrm{~L}_{12} \mathrm{~T}_{3}$ & 8247.38 & 18 & 4461.88 & 36 \\
\hline $\mathrm{L}_{4} \mathrm{~T}_{4}$ & 8389.48 & 15 & 4885.98 & 30 & $\mathrm{~L}_{12} \mathrm{~T}_{4}$ & 8091.42 & 25 & 6632.28 & 6 \\
\hline
\end{tabular}

" This is low nitrogen application trial. In this trial, no nitrogen was applied to provide the abiotic stress of low soil fertility

criterion and ranking of parents and crosses in maize for ten traits under normal and ESM conditions). Among the crosses $\mathrm{L}_{6} \times \mathrm{T}_{4}$ ranked highest and the least performing cross for this particular selection index was $\mathrm{L}_{5} \times \mathrm{T}_{1}$.

Economic weights assigned to various traits under low-N and ESM conditions in the present study have been presented in as indicated in Table 4 (Table 4: Simultaneous selection indices for normal and low-N trials in maize) and Table 5 (Table 5: Simultaneous selection indices for normal and ESM trials in maize), respectively.

Among the parents, none of the parents ranked high among all genotypes according to the assigned selection criteria in normal low-N and ESM conditions. Among the crosses aggregate score were higher in most of the cases but general trend was that ESM traits had the lower score values. Crosses $\mathrm{L}_{11} \times \mathrm{T}_{4}, \mathrm{~L}_{8} \times \mathrm{T}_{1}, \mathrm{~L}_{11} \times \mathrm{T}_{3}, \mathrm{~L}_{7} \times \mathrm{T}_{2}$, and $\mathrm{L}_{6} \times \mathrm{T}_{2}$ performed well in both normal and low-N conditions for the assigned selection criteria while crosses $\mathrm{L}_{5} \times \mathrm{T}_{4}$, and $\mathrm{L}_{12} \times \mathrm{T}_{2}$ performed poorly in low-N conditions. In ESM trials, $\mathrm{L}_{6} \times \mathrm{T}_{4}, \mathrm{~L}_{7} \times \mathrm{T}_{2}$, and $\mathrm{L}_{4} \times \mathrm{T}_{1}$ performed excellent while crosses $\mathrm{L}_{5} \times \mathrm{T}_{1}, \mathrm{~L}_{12} \times \mathrm{T}_{2}, \mathrm{~L}_{5} \times \mathrm{T}_{4}$, and $\mathrm{L}_{3} \times \mathrm{T}_{3}$ ranked very low in ESM trials. Now for ESM tolerance we can deduce from the results that crosses $\mathrm{L}_{6} \times \mathrm{T}_{4}$ and $\mathrm{L}_{7} \times \mathrm{T}_{2}$ are the best available crosses according to the given selection

Table 4: Simultaneous selection indices for normal and low-N trials in maize

\begin{tabular}{lccc}
\hline Variable/character & Economic & \multicolumn{2}{c}{$\beta$ i value } \\
\cline { 3 - 4 } & weight & Normal & ESM $^{*}$ \\
\hline ASI & 2 & -21.83 & 34.04 \\
Yield $\left(\mathrm{kg} \mathrm{ha}^{-1}\right)$ & 5 & 4.87 & 4.90 \\
Leaf senescence & 1 & -56.02 & 14.47 \\
Number of ears plant $^{-1}$ & 1 & 190.13 & 175.18 \\
\hline
\end{tabular}

* This is excess soil moisture trials. In this trial, waterlogging treatment was given at knee high growth stage for 6 days by keeping continues submergence with an average depth of ponding of about $5 \mathrm{~cm}$. After 6 days of ponding, water was drained out of the plots.
Table 5: Simultaneous selection indices for normal and ESM trials in maize

\begin{tabular}{lccc}
\hline Variable/character & Economic & \multicolumn{2}{c}{$\beta$ i value } \\
\cline { 3 - 4 } & weight & Normal & ESM \\
\hline Days to 50\% tasseling & 1 & 4.331 & 49.222 \\
Days to 50\% silking & 1 & -13.752 & -48.945 \\
ASI & 0.7 & -23.832 & 50.842 \\
Plant height & 1 & 2.325 & 0.897 \\
Ear height & 1 & 4.011 & 2.782 \\
Cob length & 1 & 29.787 & -3.385 \\
Cob diameter & 1 & -91.623 & 11.315 \\
100 kernel weight & 1 & 22.626 & 1.437 \\
Yield (kg ha ${ }^{-1}$ ) & 1.6 & 1.512 & 1.556 \\
Nodes bearing & 1.3 & -36.061 & 14.192 \\
adventitious roots & & & \\
\hline
\end{tabular}

criteria. For low-N tolerance, $\mathrm{L}_{11} \times \mathrm{T}_{4}$ and $\mathrm{L}_{8} \times \mathrm{T}_{1}$ were the best crosses. Similar studies with different objectives were conducted by Brim et al. (1959), Mulamba and Mock (1978), Kauffmann and Dudley (1979), Nawar et al. (1991) Banziger and Lafitte (1997), Edmeades et al. (1999); Modaressi et al. (2004); Kebede (2007); Lone (2006) in different stress conditions.

\section{Conclusion}

It is desirable to develop maize cultivars with increased resistance to abiotic stress for sustainable production. For this, selection indices are considered as an aid for simultaneous selection of multiple traits which can help the breeder in spotting the desirable genotype/family of a crop species in a population improvement program. The study carried out illustrates how to construct selection indices with regard to low $\mathrm{N}$ and ESM tolerance. Thus, it has been recognized that most rapid improvement in the economic value is expected from selection applied simultaneously to all the characters which determine the economic value of a plant, provided 
appropriate weights are assigned to each character.

\section{References}

Banziger, M., Lafitte, H.R., 1997. Efficiency of secondary traits for improving maize for low-nitrogen target environments. Crop Science 37, 1110-1117.

Bernardo, R., Yu. J., 2007. Prospects for genome wide selection for quantitative traits in maize online publication. Crop Science 47, May-June, 2007.

Brim, C.A., Johnson, H.W., Cockerham, C.C., 1959. Multiple selection criteria in soybeans. Agronomy Journal 51, 42-46.

CIMMYT, 1994. World maize facts and trends. Maize seed industry revisited: Emerging roles of the public and private sectors. Centro International de Mejoramiento de Maiz y Trigo (CMMYT), Mexico.

CIMMYT, 1995. International maize testing report, 1995. Centro International de Mejoramiento de Maiz y Trigo (CMMYT), Mexico.

CIMMYT, 1996. CIMMYT International maize testing report, 1996. Centro International de Mejoramiento de Maiz y Trigo (CMMYT), Mexico.

DMR, 2001. Annual report, Directorate of Maize Research (DMR), 49 ${ }^{\text {th }}$ Annual Maize Workshop, Directorate of Maize Research, held at C.S. Azad University of Agriculture and Technology, Kanpur (Uttar Pradesh), India 5-9, April 2001.

Dolan, D.J., Stuthman, D.D., Kolb, F.L., Hewings, A.D., 1996. Multiple traits selection in a recurrent selection population in Oat (Avena sativa L.). Crop Science 36(5), 1207-1211.

Edmeades. G.O., Bolanos, J., Chapman, S.C., Lafitte, H., Banziger, M., 1999. Selection improves drought tolerance in tropical maize population: I. Gains in biomass, grain yield, and harvest index. Crop Science 39(5), 1306-1315.

FAO. 2010. The state of food insecurity in the world. Addressing food insecurity in protracted crisis. Available at http://www.fao.org/publications/sofi /en/ (verified 10 Dec. 2011). Food and Agriculture Organization of the United Nations, Rome, Italy.

Hazel, L.N., 1943. Genetic basis for constructing selection indices. Genetics 28, 476-490.
Katyal, J.C., 1994. Nutrient management in soils of arid and semi-arid regions 79-91. In soil management for sustainable agriculture in Dryland Areas, Bulletin Number 16. Indian Society of Soil Science.

Kauffman, K.D., Dudley, J.W., 1979. Selection indexes for corn grain yield, \% protein and kernel weight. Crop Science $19,583-588$.

Kebede, S.A., 2007. Genetic studies on low nitrogen tolerance in maize (Zea mays L.). Ph.D. Thesis, G.B. Pant University of Agriculture and Technology, Pantnagar, India 52-53.

Lone, A. A., 2006. Genetic studies on excess soil moisture tolerance in maize (Zea mays L.). Ph.D. Thesis, G.B. Pant University of Agriculture and Technology, Pantnagar, India 107-109.

Modaressi, M., Kheradnam, M., Assad, M.T., 2004. Selection indices as indirect selection in corn hybrids (Zea mays L.) for increasing yield. Iranian Journal of Agriculture Science 35 (1), 115-127.

Mulamba, N.N., Mock, J.J., 1978. Improvement of yield potential of the Eto Blanco maize (Zea mays L.) population by breeding for plant traits. Egyptian Journal of Genetics and Cytology 7, 40-41.

Nawar, A.A., Ibrahim, M.E., Khalil, A.N.M., 1991. The efficiency of three conventional selection indices in corn. Annals of Agriculture Science 29(1), 63-75.

SAA, 2002. Feeding the future-African's crops hunger for improved nutrition. Sasakawa Africa Association Newsletter 18, 4-5.

Savita, U.S., Rathore, T.K., Mishra, H.S., 2004. Response of some maize genotypes to temporary waterlogging. Journal of Plant Biology 3(1), 29-36.

Shimizu, N., 1992. Corn cultivation in converted paddy fields in Japan. Extension Bulletin ASPA-Food and Water Centre 319-315.

Smith, H.F., 1936. A discriminant function of plant selection. Eugenics 7, 240-250.

Vikram, A., Roy, D., 2003. Selection of characters for constructing selection index in groundnut ( $A$. hypogea L.). Legume Research 26(2), 137-139.

Xie, C., Xu, S., Mosjidis, J.A., 1998. Multistage selection indices for maximum genetic gain and economic efficiency in red clover. Euphytica 98(1-2), 75-82. 